\title{
Que savons-nous des mesures visant à améliorer la couverture vaccinale?
}

\author{
Naus $\mathbf{M}^{1^{*}}$ \\ ${ }^{1}$ Centre de contrôle des maladies de la Colombie-Britannique et École de la santé publique et des populations, Université de \\ la Colombie-Britannique, Vancouver (Colombie-Britannique) \\ *Correspondance : monika.naus@bccdc.ca
}

\section{Résumé}

Au cours des cent dernières années, un nombre de plus en plus élevé de vaccins ont été mis sur le marché canadien et, pourtant, leur utilisation optimale dépend de la demande du public et de son acceptation de ces produits. Dans les années 1990, la recherche portait plus particulièrement sur les principaux obstacles à la prise vaccinale, soulignant l'importance de ces barrières à l'accessibilité et des " occasions de vaccination ratées ». $\mathrm{Au} X X I^{e}$ siècle, l'accent porte sur la réticence affichée à l'égard de la vaccination, laquelle est influencée par des facteurs tels que la complaisance, la commodité et la confiance. Ce phénomène n'est pas nouveau, mais certains de ses vecteurs le sont, y compris un calendrier d'immunisation de plus en plus chargé, des préoccupations de société de plus en plus grandes, concernant les risques par rapport aux bienfaits, et une hausse du consumérisme en matière de santé. Comprendre et aborder la réticence face aux vaccins sont des éléments essentiels si on veut éviter de compromettre le succès de la vaccination dans l'avenir. Si des recherches plus poussées s'imposent, tant des ressources visant à favoriser le dialogue entre les praticiens et des parents hésitants que des mesures visant à faire face à la réticence de la population et de la société en matière de vaccination sont nécessaires.

\section{Introduction}

La décision parentale de ne pas faire vacciner les enfants est considérée comme un obstacle croissant à la réussite des programmes d'immunisation au Canada. À la suite de l'élimination de la rougeole et de la rubéole au Canada (1) sont apparues les difficultés liées à l'introduction du vaccin contre le VPH (2), le dossier très peu flatteur sur les taux de couverture vaccinale au Canada préparé par I'UNICEF (3), une surveillance provinciale indiquant une tendance croissante du refus de la vaccination (4) et la réapparition de la rougeole (5). Tous ces éléments ont permis de faire la lumière sur le fait que la réticence face à la vaccination contribue grandement aux taux que nous connaissons en matière de prise vaccinale. Ce phénomène n'est pas nouveau, mais certains de ses vecteurs le sont, y compris un calendrier d'immunisation de plus en plus chargé, des préoccupations de société de plus en plus grandes concernant les risques par rapport aux bienfaits, et une hausse du consumérisme en matière de santé. Comprendre et aborder la réticence face aux vaccins sont des éléments essentiels si on veut éviter de compromettre le succès de la vaccination dans l'avenir. L'objectif de cet article est de résumer la littérature disponible sur les stratégies visant à contrer la réticence face à la vaccination dans un effort d'amélioration de la confiance du public et, conséquemment, de l'acceptation du vaccin.

Au cours des cent dernières années, les scientifiques et les universitaires, l'industrie du vaccin et les organismes de réglementation ont mis sur le marché canadien tout un éventail de vaccins pour la prévention primaire de maladies graves. Depuis les années 1960, le Comité consultatif national de l'immunisation (CCNI) a formulé des recommandations sur leur utilisation (6). Les ministères de la Santé provinciaux et territoriaux ont adopté ces recommandations dans le cadre de leurs programmes d'immunisation financés par les deniers publics. Par conséquent, le principal objectif des programmes d'immunisation, en matière de santé publique, est d'atteindre de hauts niveaux de prise vaccinale au sein de la population afin de maximiser les bienfaits de cette mesure de prévention (7).

Dans les années 1990, les importantes éclosions de rougeole précédant l'introduction d'une deuxième dose de vaccin dans le programme d'immunisation de routine des enfants ont mené à des travaux de recherche sur les obstacles à la prise vaccinale. Provenant essentiellement des États-Unis, ces études formaient un ensemble de 
travaux sur l'importance des occasions ratées, en matière de vaccination. En effet, il a été démontré que la sous-vaccination des enfants ayant consulté un médecin qui avait négligé de profiter de cette visite pour offrir le vaccin était l'une des principales causes de ces éclosions. Des recommandations visant à améliorer les interventions des prestataires de soins de santé ont été mises au point et plusieurs examens systématiques ont été effectués au Canada et États-Unis afin de contribuer à l'intégration de stratégies dont l'efficacité avait été éprouvée, dans les directives de prestation de services $(8,9,10,11)$. Ces stratégies ont été bien résumées par le Groupe de travail sur les services de prévention communautaires et comprennent des systèmes de rappel, des exigences en matière de vaccination, ainsi que des programmes pour les garderies, les écoles et les collèges, des visites à domicile, des systèmes d'information sur l'immunisation, des incitatifs pour les patients et les familles et l'évaluation et la rétroaction du prestataire de soins (12). Plus récemment, des techniques de réduction de la douleur fondées sur des données factuelles ont été intégrées aux lignes directrices en matière de pratique de l'immunisation afin de réduire les raisons pour lesquelles les gens choisissent de ne pas être inoculés (13).

\section{Analyse}

La littérature portant sur les facteurs parentaux associés à la prise vaccinale contient plusieurs études sur les connaissances, les attitudes et les comportements à l'égard de la vaccination; en outre, on a tenté de définir les caractéristiques des personnes et des populations qui s'opposent à la vaccination (14). Les termes « réticence face à la vaccination » sont entrés dans l'usage pour décrire les attitudes et les croyances qui pourraient interférer avec l'acception d'un vaccin ou plus, y compris les demandes des parents concernant la possibilité de mettre en place un calendrier de vaccination différent. La réticence face à la vaccination est associée à tout un éventail de comportements liés à la prise vaccinale, qu'il s'agisse de l'acceptation du vaccin malgré les doutes, la vaccination sélective, la vaccination retardée et le refus catégorique de se faire vacciner. Le Groupe consultatif stratégique d'experts (GCSE) sur l'immunisation a défini la réticence comme étant : « [...] un délai dans l'acceptation ou le refus du vaccin, malgré la disponibilité des services de vaccination. La réticence face à la vaccination est complexe et son contexte, spécifique, variant selon le moment, l'endroit et les vaccins. Elle est influencée par des facteurs tels que la complaisance, la commodité et la confiance» (15).

La réticence face à la vaccination est reconnue comme un problème mondial qui a incité l'Organisation mondiale de la Santé à favoriser l'adoption d'une approche mieux organisée, pour contrer ce phénomène $(16,17)$. La réticence est étroitement liée à la confiance du public à l'égard des vaccins, laquelle n'est pas seulement due à des enjeux de nature scientifique, mais également à des facteurs psychologiques, au milieu socioculturel, aux inclinaisons philosophiques, comme la préférence pour les solutions « naturelles », et aux questions religieuses et politiques, y compris la méfiance à l'égard des gouvernements et de l'industrie pharmaceutique. Si la mise en place d'un nouveau programme d'immunisation a toujours soulevé des préoccupations liées au contexte scientifique de la recherche sur le vaccin et aux infrastructures pour l'administrer, une attention insuffisante a été portée sur les différents facteurs qui influencent l'acceptation des différents vaccins au sein de la population (18).

Plusieurs examens de la littérature portant sur la réticence face à la vaccination ont été publiés et un rapport soulignant l'importance d'une stratégie axée sur des éléments probants a été dévoilé par le GCSE, à la suite de sa réunion du mois d'octobre $2014(15,19,20,21,22)$. Si la littérature présente un ensemble hétérogène d'approches, de populations et de résultats, le GCSE soutient la mise en œuvre d'interventions à plusieurs volets, mais bien intégrées, qui comprennent les médias de masse, la mobilisation sociale à différents niveaux et des interventions axées sur le dialogue, en plus de l'application des stratégies efficaces identifiées antérieurement (par ex., les rappels). Le GCSE a également conclu qu'il faut mettre de l'avant des recherches plus poussées, ayant un caractère formatif et conçues pour recueillir des preuves plutôt que de tester les présomptions.

\section{Stratégies à l'intention des praticiens}

Des ressources ont été mises à la disposition des praticiens pour les aider à amorcer le dialogue difficile avec les parents réticents. La présentation de preuves contribue à lancer la discussion à l'aide d'une déclaration présumant que l'enfant sera immunisé (une approche favorisant l'adhésion), laquelle reconnaît que les parents perçoivent cette décision comme étant complexe et émotive. À partir d'observations sur l'« architecture de choix ", dans de telles situations, les êtres humains choisiront une décision qui a déjà été prise par la majorité (23). Selon les lignes directrices disponibles, il est recommandé d'écouter le point de vue et les préoccupations 
des parents sans porter de jugement, et on insiste sur l'importance d'établir la confiance. Les entrevues motivationnelles, effectuées à l'aide de questions ouvertes et en adoptant un style bienveillant, sont recommandées pour connaître les motivations des parents, déterminer s'ils sont ouverts au changement et établir leur position parmi les cinq étapes du modèle transthéorique de changement (précontemplation, contemplation, préparation, action, maintien) (24).

Les lignes directrices en matière de pratique clinique élaborées par des experts indiquent que le dialogue devrait également permettre de répondre à des préoccupations sur de l'information que les parents auraient lue ou entendue $(25,26,27)$. La littérature suggère que l'information écrite sur la vaccination et le fait de réfuter les conceptions erronées peuvent renforcer les convictions des parents les plus farouchement opposés à la vaccination (28). Les preuves soulignent l'importance de mettre en valeur les bienfaits de la vaccination pour l'enfant, au lieu de parler des avantages pour la société dans son ensemble (29). Des exemples d'histoires sur des cas de maladies qu'on peut prévenir à l'aide de la vaccination peuvent s'avérer plus utiles que le fait d'énumérer des statistiques, mais il est important de définir la terminologie numérique utilisée (par exemple " courant » ou « rare ») pour décrire le risque de maladies et leurs complications, ainsi que la fréquence des événements défavorables.

La rencontre est plus efficace lorsque l'information fournie aux parents correspond à leurs préoccupations. Les prestataires de soins de santé devraient être bien informés afin de répondre aux questions des parents. La recherche démontre en effet que des réponses vagues ne favorisent pas l'établissement de la confiance. Une excellente série d'articles est offerte dans Pédiatriques (30,31,32, 33). Les prestataires de services devraient s'efforcer de ne pas trop « vendre " l'immunisation, de souligner les attentes et la gestion des inconvénients les plus courants, y compris les réactions au lieu de l'injection et la fièvre chez les nourrissons et les jeunes enfants, d'aborder la crainte des parents relativement à la douleur associée aux vaccins injectables et d'offrir des moyens de la réduire (13). Le prestataire devrait formuler des recommandations claires, puisqu'il a été constaté à maintes reprises qu'elles sont étroitement liées à l'acceptation de la vaccination par les parents. La plupart de ces renseignements ont été résumés au sein de ressources disponibles en ligne et peuvent être facilement accessibles et remis aux parents $(34,35,36)$.

\section{Stratégies du programme}

À plus large échelle, des stratégies valent la peine d'être explorées. Elles comprennent le recours à des personnes qualifiées ainsi qu'à séances de groupe avec les parents, avant le début de la vaccination (37), et une réponse en temps opportun des autorités en santé publique, lors de rapports négatifs dans les médias ou de travaux scientifiques bâclés $(38,39)$. La mobilisation d'un plus grand nombre de personnes dans la défense de la vaccination est une stratégie émergente prometteuse qui devra être évaluée $(40,41)$.

\section{Conclusion}

Malgré le fait que les vaccins occupent la deuxième place après l'eau potable en matière de stratégies visant à sauver des vies sur la planète, il n'y a pas de recette magique pour favoriser leur acceptation (42). Pour assurer le succès continu de ces programmes, il est important de concentrer notre attention sur les préoccupations de la population, de la collectivité et des individus, afin de mieux comprendre où elles se situent dans le continuum de l'acception au rejet. Cette réponse et les approches à plusieurs volets conçues pour des communautés et des vaccins en particulier sont essentielles si on veut améliorer l'accueil réservé par le public à ces vaccins et arriver non seulement à améliorer la prise vaccinale, mais à assurer une plus grande confiance.

\section{Remerciements}

J'aimerais remercier tous les collègues des domaines de la santé et de la pédiatrie pour leur engagement dans la prestation de programmes d'immunisation de qualité.

\section{Conflit d'intérêts}

\section{Aucun}




\section{Financement}

Aucun

\section{Références}

(1) Public Health Agency of Canada. Elimination of Measles, Rubella and Congenital Rubella Syndrome in Canada: Documentation and Verification Report. Executive Summary. 2013 Apr 4. http://www.phac-aspc.gc.ca/im/vpd-mev/measles-rougeole-mrer-eng.php (disponible en français: http://www.phacaspc.gc.ca/im/vpd-mev/measles-rougeole-mg-fra.php).

(2) Ogilvie G, Anderson M, Marra F, McNeil S, Pielak K, Dawar M, Mclvor M, Ehlen T, Dobson S, Money D, Patrick DM, Naus M. A population-based evaluation of a publicly funded, school-based HPV vaccine program in British Columbia, Canada: Parental factors associated with HPV vaccine receipt. PLoS Med. 2010 May 4;7(5):e1000270.

(3) UNICEF Office of Research. Child well-being in rich countries: A comparative overview. Innocenti Report Card 11. Florence: UNICEF Office of Research; 2013. http://www.unicef-irc.org/publications/pdf/rc11_eng.pdf

(4) Canadian Immunization Conference 2014. Speaker Presentations. Programs Panel Session 1. Tuesday December 2, 11:00 am, Room 210. Immunization Coverage in Canada. Presentations by Samara David (British Columbia) and Sarah Wilson (Ontario). http://cic2014.isiglobal.ca/calendar/1

(5) Public Health Agency of Canada (PHAC). The Chief Public Health Officer's Report on the State of Public Health in Canada 2013: Infectious Disease-The Never-ending Threat. Ottawa: PHAC; 2013. Page 9. http://www.phac-aspc.gc.ca/cphorsphc-respcacsp/2013/assets/pdf/2013-eng.pdf (disponible en français: http://www.phac-aspc.gc.ca/cphorsphc-respcacsp/2013/assets/pdf/2013-fra.pdf).

(6) Public Health Agency of Canada. Canadian Immunization Guide. 2014 Apr 23. http://www.phac-aspc.gc.ca/publicat/cig-gci/index-eng.php (disponible en français: http://www.phacaspc.gc.ca/publicat/cig-gci/index-fra.php).

(7) Public Health Agency of Canada. Immunization Coverage in Canada (2002 to 2012). 2015 Jan 30. http://www.phac-aspc.gc.ca/im/nics-enva/icc-cvc-eng.php (disponible en français: http://www.phac-aspc.gc.ca/im/nics-enva/icc-cvc-fra.php).

(8) Gyorkos TW, Tannenbaum TN, Abrahamowicz M, Bédard L, Carsley J, Franco ED, Delage G, Miller MA, Lamping DL, Grover SA. Evaluation of the effectiveness of immunization delivery methods. Can J Public Health. 1994 Jul-Aug;85 Suppl 1:S14-30.

(9) Tannenbaum TN, Gyorkos TW, Abrahamowicz M, Bédard L, Carsley J, Franco ED, Delage G, Miller MA, Lamping DL, Grover SA. Immunization delivery methods: Practice recommendations. Can J Public Health. 1994 Jul-Aug;85 Suppl 1:S37-4.

(10) Briss PA, Rodewald LE, Hinman AR, Shefer AM, Strikas RA, Bernier RR, Carande-Kulis VG, Yusuf HR, Ndiaye SM, Williams SM. Reviews of evidence regarding interventions to improve vaccination coverage in children, adolescents, and adults. The Task Force on Community Preventive Services. Am J Prev Med. 2000 Jan;18(1 Suppl):97-140.

(11) Task Force on Community Preventive Services. Recommendations Regarding Interventions to Improve Vaccination Coverage in Children, Adolescents, and Adults. Am J Prev Med. 2000;18(1S):92-6.

(12) Community Preventive Services Task Force. Topic: Vaccination-Increasing Appropriate Vaccination. Community Guide Branch. Division of Public Health Information Dissemination. Center for Surveillance, Epidemiology and Laboratory Services. Office of Public Health Scientific Services. Centers for Disease Control and Prevention. 2015 Feb 13. http://www.thecommunityguide.org/vaccines/index.html

(13) Taddio A, Appleton M, Bortolussi R, Chambers C, Dubey V, Halperin S, Hanrahan A, Ipp M, Lockett D, MacDonald N, Midmer D, Mousmanis P, Palda V, Pielak K, Riddell RP, Rieder M, Scott J, Shah V. Reducing the pain of childhood vaccination: An evidence-based clinical practice guideline. CMAJ. 2010 Dec14;182(18):E843-55.

(14) Jacobson RM, Targonski PV, Poland GA. A taxonomy of reasoning flaws in the anti-vaccine movement. Vaccine. 2007 Apr 20;25(16):3146-52.

(15) Report of the SAGE Working Group on Vaccine Hesitancy. 2014 Nov 12. http://www.who.int/immunization/sage/meetings/2014/october/SAGE_working_group_revised_report_vaccine_hesita ncy.pdf?ua $=1$

(16) Streefland P, Chowdhury AM, Ramos-Jimenez P. Patterns of vaccination acceptance. Soc Sci Med. 1999 Dec;49(12):1705-16. 
(17) Dubé E, Gagnon D, Nickels E, Jeram S, Schuster M. Mapping vaccine hesitancy-Country-specific characteristics of a global phenomenon. Vaccine. 2014 Nov 20;32(49):6649-54.

(18) Larson HJ, Cooper LZ, Eskola J, Katz SL, Ratzan S. Addressing the vaccine confidence gap. Lancet. 2011 Aug 6;378(9790):526-35.

(19) Dubé E, Laberge C, Guay M, Bramadat P, Roy R, Bettinger J. Vaccine hesitancy: An overview. Hum Vaccin Immunother. 2013 Aug;9(8):1763-73.

(20) Larson HJ, Jarrett C, Eckersberger E, Smith DM, Paterson P. Understanding vaccine hesitancy around vaccines and vaccination from a global perspective: A systematic review of published literature, 2007-2012. Vaccine. 2014 Apr 17;32(19):2150-9.

(21) Gowda C, Dempsey AF. The rise (and fall?) of parental vaccine hesitancy. Hum Vaccin Immunother. 2013 Aug;9(8):1755-62.

(22) Sadaf A, Richards JL, Glanz J, Salmon DA, Omer SB. A systematic review of interventions for reducing parental vaccine refusal and vaccine hesitancy. Vaccine. 2013 Sep 13;31(40):4293-304.

(23) Opel DJ, Omer SB. Measles, mandates, and making vaccination the default option. JAMA Pediatr. 2015 Feb 11. doi: 10.1001/jamapediatrics.2015.0291. [Epub ahead of print] PubMed PMID: 25671505.

(24) Leask J, Kinnersley P, Jackson C, Cheater F, Bedford H, Rowles G. Communicating with parents about vaccination: A framework for health professionals. BMC Pediatr. 2012 Sep 21;12:154.

(25) Halperin S. How to manage parents unsure about immunization. Canadian Journal of CME. 2000 Jan:62-75. www.path.org/vaccineresources/details.php?i=152

(26) MacDonald NE, Finlay JC, Canadian Paediatric Society Infectious Diseases and Immunization Committee. Working with vaccine-hesitant parents. Paediatr Child Health. 2013;18(5):265-7.

http://www.cps.ca/documents/position/working-with-vaccine-hesitant-parents

(27) Healy CM, Pickering LK. How to communicate with vaccine-hesitant parents. Pediatrics. $2011 \mathrm{May}$; 127 Suppl 1:S127-33.

(28) Nyhan B, Reifler J, Richey S, Freed GL. Effective messages in vaccine promotion: A randomized trial. Pediatrics. 2014 Apr;133(4):e835-42.

(29) Hendrix KS, Finnell SM, Zimet GD, Sturm LA, Lane KA, Downs SM. Vaccine message framing and parents' intent to immunize their infants for MMR. Pediatrics. 2014 Sep;134(3):e675-83.

(30) Offit PA, Moser CA. The problem with Dr Bob's alternative vaccine schedule. Pediatrics. 2009 Jan;123(1):e164-9.

(31) Offit PA, Jew RK. Addressing parents' concerns: Do vaccines contain harmful preservatives, adjuvants, additives, or residuals? Pediatrics. 2003 Dec;112(6 Pt 1):1394-7.

(32) Offit PA, Hackett CJ. Addressing parents' concerns: Do vaccines cause allergic or autoimmune diseases? Pediatrics. 2003 Mar;111(3):653-9.

(33) Offit PA, Quarles J, Gerber MA, Hackett CJ, Marcuse EK, Kollman TR, Gellin BG, Landry S. Addressing parents' concerns: Do multiple vaccines overwhelm or weaken the infant's immune system? Pediatrics. 2002 Jan;109(1):124-9.

(34) Derban A, Harper J, Jiwa S, et al. ImmunizeBC. Quick Reference: Immunization Communication Tool for Immunizers. 2013.

http://www.immunizebc.ca/sites/default/files/docs/ict_final.pdf

(35) Centers for Disease Control and Prevention, American Academy of Family Physicians, American Academy of Pediatrics. Provider Resources for Vaccine Conversations with Parents. 2014 Dec 17.

http://www.cdc.gov/vaccines/hcp/patient-ed/conversations/index.html

(36) Canadian Paediatric Society. Your Child's Best Shot: A parent's guide to vaccination. 4th ed. Moore DL, editor. 2015. https://bookstore.cps.ca/stock/details/your-childs-best-shot-a-parents-guide-to-vaccination-4th-edition

(37) Jackson C, Cheater FM, Harrison W, Peacock R, Bekker H, West R, Leese B. Randomised cluster trial to support informed parental decision-making for the MMR vaccine. BMC Public Health. 2011 Jun 16;11:475.

(38) Nicholson MS, Leask J. Lessons from an online debate about measles-mumps-rubella (MMR) immunization. Vaccine. 2012 May 28;30(25):3806-12.

(39) Centers for Disease Control and Prevention. Clinical Immunization Safety Assessment (CISA) Network Publications and Technical Reports. Technical Reports. Review of a published report of cerebral vasculitis after vaccination with the Human Papillomavirus (HPV) Vaccine. 2012 Nov 9.

http://www.cdc.gov/vaccinesafety/Activities/cisa/technical_report.html

(40) Within Reach. What can parents do to support immunization? Seattle, WA; 2015 Feb 13. http://www. withinreachwa.org/what-can-parents-do-to-support-immunization/

(41) I Boost Immunity, BC. Sponsored by the Public Health Association of BC. 
http://www.iboostimmunity.ca/

Andre FE, Booy R, Bock HL, Clemens J, Datta SK, John TJ, Lee BW, Lolekha S, Peltola H, Ruff TA, Santosham M, Schmitt HJ. Vaccination greatly reduces disease, disability, death and inequity worldwide. Bull World Health Organ. 2008 Feb;86(2):140-6. 